\title{
ULTRA-HIGH ENERGY COSMIC RAY PRODUCTION BY CURRENT DISRUPTION IN ACTIVE GALACTIC NUCLEI
}

\author{
George Lake \\ Bell Laboratories \\ R. E. Pudritz \\ Astronomy Dept., University of California
}

\begin{abstract}
Electrodynamic models for the activity of galactic nuclei are shown to be current systems which can be examined in terms of equivalent circuits. The resulting inductive circuit which describes the coupling of the generator (black-hole and accretion disk) to the distant load (jet plasma) is prone to various instabilities. We consider the disruption of this current system and propose that ultraenergetic cosmic rays $\left(E \sim 10^{19}-10^{21} \mathrm{eV}\right)$ could be produced during the discharges, which occur at distances of $\sim 10^{16}-10^{18} \mathrm{~cm}$ from the central massive hole $\left(M \sim 10^{8} M_{\odot}\right)$. Such discharges will also produce variable $\gamma$-ray and X-ray activity and we discuss observations of Cen $\mathrm{A}$ in this regard.
\end{abstract}

\section{INTRODUCTION}

Current observations of ultra-high energy cosmic rays $\left(E>10^{18} \mathrm{eV}\right)$ show an "ankle" in the spectrum at $E \geqslant 10^{19} \mathrm{eV}$ (Cunningham et al. 1980) with $\mathrm{n}(\mathrm{E})=3.1 \times 10^{12} \mathrm{E}^{-2.31}$ particles $\mathrm{m}^{-2} \mathrm{eV}^{-1} \mathrm{~s}^{-1}$ and a maximum detected energy of $1.6 \times 10^{20} \mathrm{eV}$. There may be an anisotropy in arrival directions towards the Virgo cluster although this is as yet not certain. The energy density of particles of energy $>10^{19} \mathrm{eV}$ of $\sim 4 \times 10^{-8} \mathrm{eV} \mathrm{cm}^{-3}$ which needs to be replenished every $10^{16} \mathrm{~s}$ due to photopion production by microwave background photons. This yields a source energy density of $1.6 \times 10^{38} \mathrm{ergs} \mathrm{s}^{-1} \mathrm{Mpc}^{-3}$. Type 1 Seyfert galaxies qualify as potential sources as they have a spatial density of $10^{-4} \mathrm{Mpc}^{-3}$ and a $0.5-4.5 \mathrm{keV}$ output of $10^{44} \mathrm{ergs} \mathrm{s}^{-1}$ or $10^{40} \mathrm{erg} \mathrm{s}^{-1} \mathrm{Mpc}^{-3}$. If NGC4151 (Lawrence 1980) is typical then the combined $x$ and $\gamma$-ray energy densities are such that the efficiency of $\mathrm{E}>10^{19} \mathrm{eV}$ particle production need only be $10^{-3}$ or $10^{-4}$.

The relativistic jets of many active galactic nuclei have stimulated several electrodynamic models for the required production of highly collimated energetic flows (Lovelace 1976, Blandford and Znajek 1977, Blandford and Payne 1982). Potential drops in excess of $10^{19} \mathrm{eV}$ may be produced by magnetized accretion disks around massive black-holes $\left(\mathrm{M} \sim 10^{8}-10^{9} \mathrm{M}_{\odot}\right)$ at the "heart" of the engine. The electrodynamic coupling between the black-hole plus disk (generator) with the jet (load) might produce even larger potential drops during the disruption of this current system accelerating particles to $10^{21} \mathrm{eV}$ on scales of $\sim 10^{-2}-10^{-1} \mathrm{pc}$ from the central engine. We 
will describe this phenomena of current disruption using the technique of equivalent circuits, borrowed from the analogous solar and terrestrial cases (Spicer 1982, Chiu and Cornwall 1980).

\section{THE COUPLING OF ACCRETION DISK AND JET PLASMA}

It seems inevitable that accretion disks possess dynamically important magnetic fields $\left(10^{3}-10^{4}\right.$ Gauss) due either to the compression of plasma containing field (eg. Lovelace 1976) or by in situ generation by a turbulent dynamo (Pudritz and Fahlman 1982). The radial electric field generated by matter in a rotating disk $\left(E_{r}=\left(v_{\phi} / c\right) B_{p}\right)$ generates large potential drops across the disk, e.g.

$$
\mathrm{V}_{\text {gen }} \sim 10^{19}\left(\mathrm{~B} / 10^{3} \text { Gauss }\right)\left(\mathrm{M} / 10^{8} \mathrm{M}_{\odot}\right) \text { Volts }
$$

Now magnetic field lines which are tied to the disk will connect to plasma in the jet region. Imperfect coupling of disk and jet plasmas means field lines are not equipotential surfaces, (Lee and Kan 1981), so acceleration of particles (protons in our case) can occur. If the magnetic coupling of the earth's magnetospheric plasma with the ionosphere is any guide (Chui and Cornwall 1980), the global boundary conditions necessitate a description as a current system. In the terrestrial context, the auroral zone produced as a result of such coupling yields a parallel electric field $E_{\|}$, which a magnetohydrodynamic analysis cannot consistently explain.

Field lines attached to the differentially rotating disk are twisted on a time-scale $\mathrm{t}_{\mathrm{dyn}}=0.7 \times 10^{4}\left(\mathrm{M} / 10^{8} \mathrm{M}_{\odot}\right)\left(\sigma / \sigma_{\mathrm{i}}\right)^{3 / 2} \mathrm{~s}$ where $\sigma_{\mathrm{i}} \sim 10^{14}\left(\mathrm{M} / 10^{8} \mathrm{M}_{\odot}\right) \mathrm{cm}$ is the inner disk radius and $\sigma$ is the position of the footpoint. This twisting drives a current through the disk magnetosphere of magnitude

$$
I_{0}=V_{\text {gen }} / R_{0}
$$

where $R_{0}$ is the load resistance (jet plasma) to which the generator is coupled. In steady state, the power generated by the (black-hole and disk) is dissipated in the jet plasma at a rate $\mathscr{L}(\mathscr{L}$ is the observed jet luminosity);

$$
\begin{aligned}
\mathrm{R}_{\mathrm{o}} & =\mathrm{V}_{\mathrm{gen}}^{2} / \mathscr{L}=\mathrm{f}_{\text {sp.ch. }} \\
\mathrm{f} & \equiv\left(\mathrm{B} / 10^{3} \text { Gauss }^{2}\left(\mathrm{M} / 10^{8} \mathrm{M}_{\mathrm{O}}\right)^{2} /\left(\mathscr{L} / 10^{44} \text { ergs. } \mathrm{s}^{-1}\right)\right.
\end{aligned}
$$

and $R_{\text {sp.ch. }}=\mathrm{c}^{-1}=25 \mathrm{ohms}$ is the space charge limited resistance so that the current is $I_{0}=3 \times 10^{17} \mathrm{f}\left[V_{\text {gen }} / 3 \times 10^{19} \mathrm{~V}\right]$ Amps. This current system has an associated magnetic field in which energy is stored, and an inductance $\mathrm{L}_{\mathrm{o}}$ characterizing this global circuit is introduced. Because the magnetic flux of the system is large $\left(\mathrm{L}_{0} \mathrm{I}_{0}\right)$, perturbations of the magnetospheric current system will result in instability and "discharge" of this circuit converting stored magnetic energy into highenergy particle production (see Alfvén and Carlquist 1967).

The time-scale on which energy is stored is

$$
t_{\text {store }}=L_{o} / R_{0}
$$

while the voltage across the discharge region is

$$
\mathrm{V}_{\text {disch }}=\mathrm{L}_{\mathrm{o}} \mathrm{I}_{\mathrm{o}} / \mathrm{t}_{\text {disch }}=\left(\mathrm{t}_{\text {store }} / \mathrm{t}_{\text {disch }}\right) \mathrm{V}_{\text {gen }} \text {. }
$$

The time-scale to store energy in this system ought to be roughly $t_{\text {store }} \geq t_{\text {dyn }}$. The magnetic flux associated with the disk increases outwards (see Blandford and Payne 1982) so that for a disk extending to $\sigma=10-10^{2} \sigma_{\mathrm{i}}, \mathrm{t}_{\text {store }} \geq 10^{6}-10^{7} \mathrm{sec}$. Observations of x-ray variability in active galactic nuclei (Lawrence 1980) indicate discharge times of order $10^{4}-10^{5} \mathrm{~s}$, so that $\mathrm{V}_{\text {disch }} \sim 10^{2} \mathrm{~V}_{\text {gen }}$ is reasonably obtained.

We argue that some type of electrostatic field with $E_{\|} \neq 0$ developes in the discharge region as in 
the theory of double layers (e.g., Carlquist 1982) or variants (e.g., Chiu and Cornwall). A magnetically active accretion disk will have many buoyant loops of intense field visiting into the disk corona (Pudritz and Fahlman 1982) and these perturbations may be sufficient to cause the large scale magnetospheric current system to "discharge" (see Lake and Pudritz 1983 for a more detailed discussion).

\section{DISCUSSION}

An observable consequence of such a scheme for particle acceleration is the presence of intense, highly variable $\gamma$-ray activity. During discharge, cold electrons from the jet are accelerated towards the accretion disk and inverse scatter soft disk photons to produce hard $\gamma$-rays, softening towards $x$ rays at the end of the disruptive event. As an example, if the nucleus of Cen $A$ is the primary source of the observed ultra-high energy particles; the power must be $\sim 5 \times 10^{42}$ ergs s$^{-1}$ and the associated $\gamma$-ray luminsosity would be $\sim 10^{42} \mathrm{ergs} \mathrm{s}^{-1}$, with a characteristic energy of $\sim 3 \times 10^{10} \mathrm{eV}$. This compares favorably with the time-varying flux of $10^{41} \mathrm{erg} \mathrm{s}^{-1}$ seen at energies of $10^{12}-10^{14} \mathrm{eV}$ (Grindlay 1982). NGC4151 may also show such variable $\gamma$-ray activity (White et al. 1980) at lower energies (1-20 MeV) and certainly any such trend in Type I Seyferts would enhance the prospects of such a theory.

\section{ACKNOWLEDGEMENTS}

We thank J. Arons, A. Hasegawa, K. Jacobs, J. Linsley, C. McKee, J. Ostriker, and A. Watson for stimulating conversations. REP acknowledges financial support of NSF Grant AST 79-23243 to the University of California at Berkeley.

\section{REFERENCES}

[1] Alfvén, H. and Carlquist, P.: 1967, Solar Phys. 1, 220.

[2] Blandford, R. D. and Payne, D. G.: 1982, MNRAS 199, 883.

[3] Blandford, R. D. and Znajek, R. L.: 1977, MNRAS 179, 443.

[4] Carlquist, P.: 1982, Astrophys. Sp. Sc. 87, 21.

[5] Chiu, Y. T. and Cornwall, J. M.: 1980, J. Geophys. Res. 85, 543.

[6] Cunningham, G., Lloyd-Evans, J. Pollock, A., Reid, R. and Watson, A.: 1980, Ap. J. (Letters) 236, L71.

[7] Grindlay, J. E.: 1982, Proceedings of Int. Workshop on VHE Gamma Ray Astronomy (Ootacamund, India) in press.

[8] Lake, G. and Pudritz, R. E.: 1983, submitted Ap. J.

[9] Lawrence, A.: 1980, MNRAS 1982, 83.

[10] Lee, L. C. and Kan, J. R.: 1981, "Physics of Auroral Arc Formation" Eds. S-I. Akasofu and J. R. Kan, American Geophys. Union.

[11] Lovelace, R. V. E.: 1976, Nature 262, 649.

[12] Lynden-Bell, D.: 1969, Nature 223, 690.

[13] Pudritz, R. E. and Fahlman, G. G.: 1982, MNRAS 198, 689.

[14] Spicer, O.: 1982, Sp. Sc. Rev. 31, 351.

[15] White, R. S., Dayton, B., Gibbons, R., Long, J. L., Zanrosso, F. M., and Zych, A. D.: 1980, Nature 284, 608. 